\title{
The Role of Reversed Homodigital Island Flap in Digital Reconstruction
}

\author{
KHALED M. HASSAN, M.D. \\ The Department of Plastic and Reconstructive Surgery, Faculty of Medicine, Minia University, Egypt
}

\begin{abstract}
Background: Fingertip injuries are very common. Reconstruction of extensive distal phalangeal defects with exposure of tendon, bone, or joint can be particularly a difficult problem. Options are local, regional and free flaps. SSG on bone and tendon is unsuccessful and nondurable. The primary goals of digital reconstruction are to preserve digital length and maintain full mobility of the digit with providing adequate protective cover of the deeper vital structures with soft tissue and skin of good quality. Homodigital artery island flap has been found to be very useful and is commonly used for the recon $\neg$ struction of fingertip amputations.
\end{abstract}

Patients and Methods: We used the reversed homodigital artery island flap to reconstruct fingertip injuries in 8 patients at Minia University Hospital and private sector in the period between January to December 2017. We retrospectively analyzed patients for: Age, sex, mechanism of injury, location of defect, flap survival, sensory recovery and donor site coverage.

Results: Five patients were males and 3 females. Age from 18 to 42 years mean (27.3 years). Six patients had complete flap survival and two patients suffered venous congestion that resolved completely by conservative measures. Donor site closure was directly closed in all cases. Near normal sensory recovery was noted.

Conclusion: The reverse homodigital island flap is a safe method for distal phalangeal reconstruction offering multiple advantages over other local, regional and free flaps with an almost negligible donor site defect. Venous congestion and inability to reconstruct proximal finger defects are drawbacks.

Key Words: Homodigital flap - Reconstruction.

\section{INTRODUCTION}

One of the most common presentations in emergency departments is fingertip injuries. This type of injury may cause long-term cosmetic and sensory consequences affecting daily activities depending on the severity of the injury. Crush injury to the fingertip causes a straight, stellate or tearing laceration of the nail bed due to compression of the nail bed between the nail and underlying bone. It may lead to amputation of the fingertip if a sharp object is involved [1].
Assessment of the affected digit involves examining for neurovascular integrity, tendon or ligament injury, soft tissue laceration or skin loss and bony tenderness. Complete examination of sensation, vascular status and motor function should also be carried out before inserting a digital block [2].

It is important to remind ourselves frequently of the aims of surgical intervention when faced with any injury of the hand requiring reconstruction. The primary goals of digital reconstruction are to preserve digital length and maintain full mobility of the digit with providing adequate protective cover of the deeper vital structures with soft tissue and skin of good quality. The skin must not only be durable but of adequate sensibility for the normal function of that part of that particular digit. Over and above these functional aspects of reconstruction, it is necessary to remember that our hands are used as organs of expression and are also an integral part of our appearance. In respect of appearance, the hand must, at very least, be sufficiently normal as to pass unnoticed. Many reconstructed hands which are considered as a source of pride to the surgeon are doomed by their appearance to remain in the patients pocket or hidden up a sleeve [3].

The restoration of normal appearance depends, not only on the static appearance and posture of the hand, but also on its ability to move with fluidity, at least within the middle part of its normal range of motion. We can often achieve more aesthetic results in our reconstructions by minor modifications of the same reconstructive techniques, while losing nothing in terms of function [4].

In attempting to restore the optimum, it is important to recognize those techniques which are likely to prevent one achieving this goal. A shortened and/or stiff digit is usually disadvantaged 
functionally and cosmetically. In respect of the appearance loss of the nail complex is a particular disadvantage, we avoid terminalisation, encourage distal replantation or composite graft replacement, and also look to homodigital reconstruction whenever possible [3].

Since movement is so important to both the function and the appearance of the digits, we avoid techniques of bandaging, splinting and skeletal fixation which prevent immediate mobilization of the injured hand. In respect of both the appearance and the mobility of digits, time-honoured plastic surgical techniques of skin cover, such as crossfinger, thenar, groin and cross-arm flaps which necessitate tethering the injured part to another part of the body, are undesirable, although occasionally necessary [4].

Among several different homodigital artery flap reconstruction methods, homodigital artery island flap has been found to be very useful and is commonly used for the reconstruction of fingertip amputations [5,6]. Homodigital artery flap reconstruction is known to provide satisfactory texture and cosmetic appearance as well as favorable sensibility [7]. The nature of these flaps allows the surgical scars to affect only one side of the finger. The dorsal surface of the finger remains intact unlike other homodigital flaps, such as the homodigital dorsal middle phalangeal neurovascular advancement flap [8] or dorsally extended digital island flap [9].

Despite the fact that homodigital artery island flap does not include digital nerves, it was reported that patients showed good sensory recovery in the long term [10]. In general, choosing to use homodigital artery island flap depends on the level of amputation, required flap size and distal amputation through the nail bed, such as the subzone II amputation [11].

In this study, we will evaluate reversed homodigital artery sensate flap in reconstruction of fingertip amputations, examining the recovery in fingertip sensation, the range of motion and the cosmetic appearance.

\section{PATIENTS AND METHODS}

This is a retrospective non randomized clinical study.

Reverse homodigital island flap was used to reconstruct distal phalangeal dorsal and pulp defects in 8 patients at Minia University Hospital and private sector in the period between January and
December 2017. All patients and/or relatives signed an informed consent.

Immune compromised patients, poly-trauma patients, thumb amputations, defects proximal to distal interphalangeal joint, proximal digital vascular injuries, heavy smokers and patients with chronic ischemia were excluded from the study.

\section{Surgical technique (Figs. 1,2):}

Preoperative digital Allen's test is routinely performed to identify the dominant digital artery which is nearer to the midline of the hand (between middle and ring fingers) in most cases.

Prophylactic antibiotics were given. Regional intravenous anesthesia, digital nerve block or GA with tourniquet was used as indicated. Measurement of size and shape of the defect was done and flap is designed along mid-lateral axis of the proximal phalanx of the same finger showing the defect.

Under loupe magnification flap is raised in a proximal to distal direction after identification of neurovascular bundle with attention not to use the dominant digital artery. We separate the digital artery from the digital nerve thus preserving the later. We make sure to preserve a good cuff of subcutaneous tissues around the vascular pedicle to allow adequate venous drainage. Nerve dissection is never carried out beyond $3-5 \mathrm{~mm}$ proximal to distal inter-phalangeal joint. As this area corresponds to the distal transverse digital artery. Flap is transferred to the defect and sutured by $5 / 0$ prolene sutures. Donor site may be skin grafted or primarily closed depending on the size of the flap.

We retrospectively analyzed patients for age, sex, mechanism of injury, location of defect, flap survival, donor site coverage, sensory distribution, occurrence of cold intolerance, length of occupational disability and joint mobility.

Range of motion was tested using the goniometer to measure total active motion (TAM). TAM was defined as the ratio of the total arc of motion in the affected digits compared with the contralateral digits, and was recorded for the distal interphalangeal (DIP), proximal interphalangeal (PIP), and metacarpophalangeal (MP) joints.

\section{RESULTS}

Five patients were males and 3 females. Age from 18 to 42 years mean (27.3 year). Four patients had crush injuries, 2 patients had avulsion injury of the pulp of distal phalanx. 2 patients painful 
parrot peak deformity of the distal phalanx after old trauma. Pulp and dorsal defects were reconstructed in 5 and 3 patients respectively (Figs. 2,3).

Indications for flap coverage were exposed bone, tendon and tip augmentation in 4, 2, 2 patients respectively. Sensate flap including digital nerve were performed in 1 patient for pulp defect.

Six patients had uneventful recovery with complete flap survival and two patients suffered

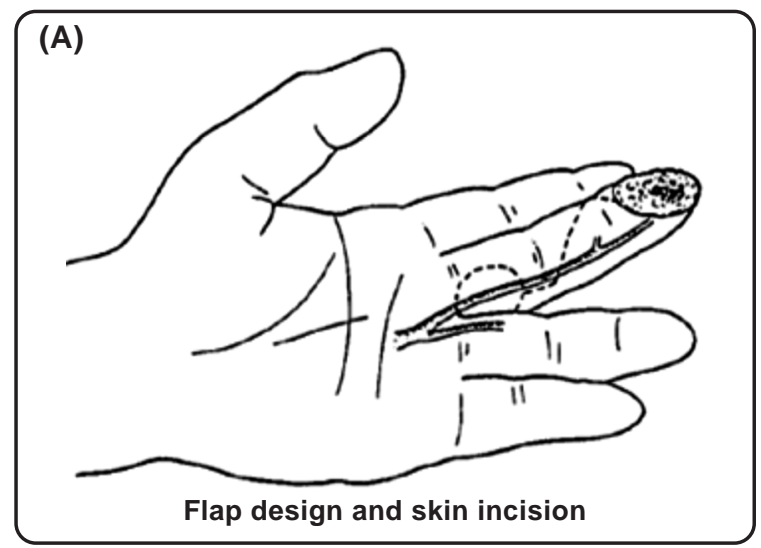

venous congestion that resolved completely on low molecular weight heparin. Donor site closure was obtained by means of direct closure in all cases.

The two point discrimination test after 6 months follow-up had a mean value of $9 \mathrm{~mm}$. None of our patients had cold intolerance. All patients returned to full activity with full range of movement within average time period of 5 weeks.

Fig. (1): Diagrammatic representation of the design, skin incision and elevation of reversed flow homodigital island flap.
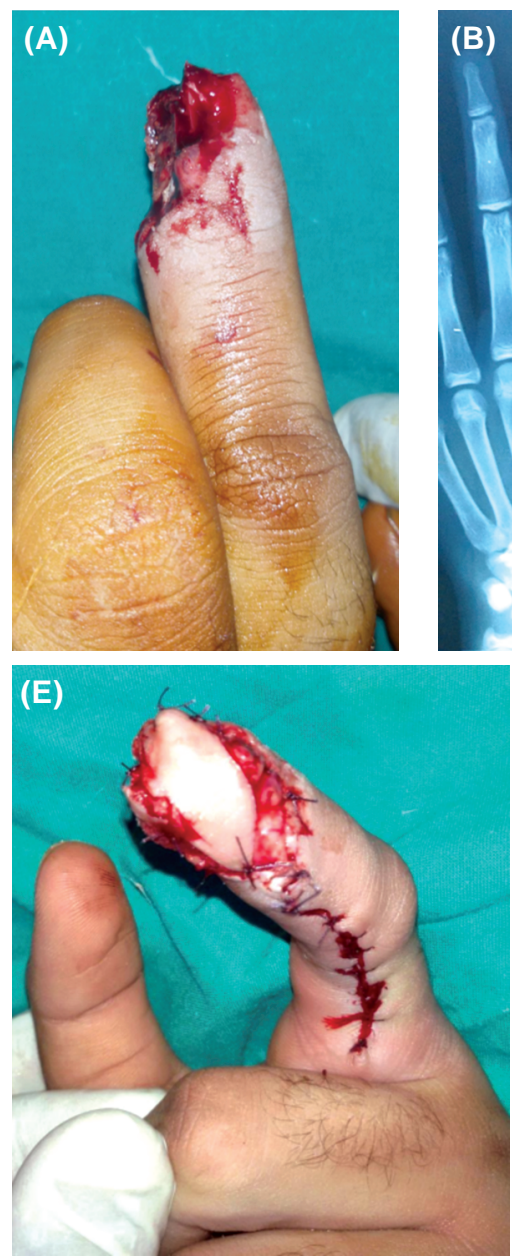
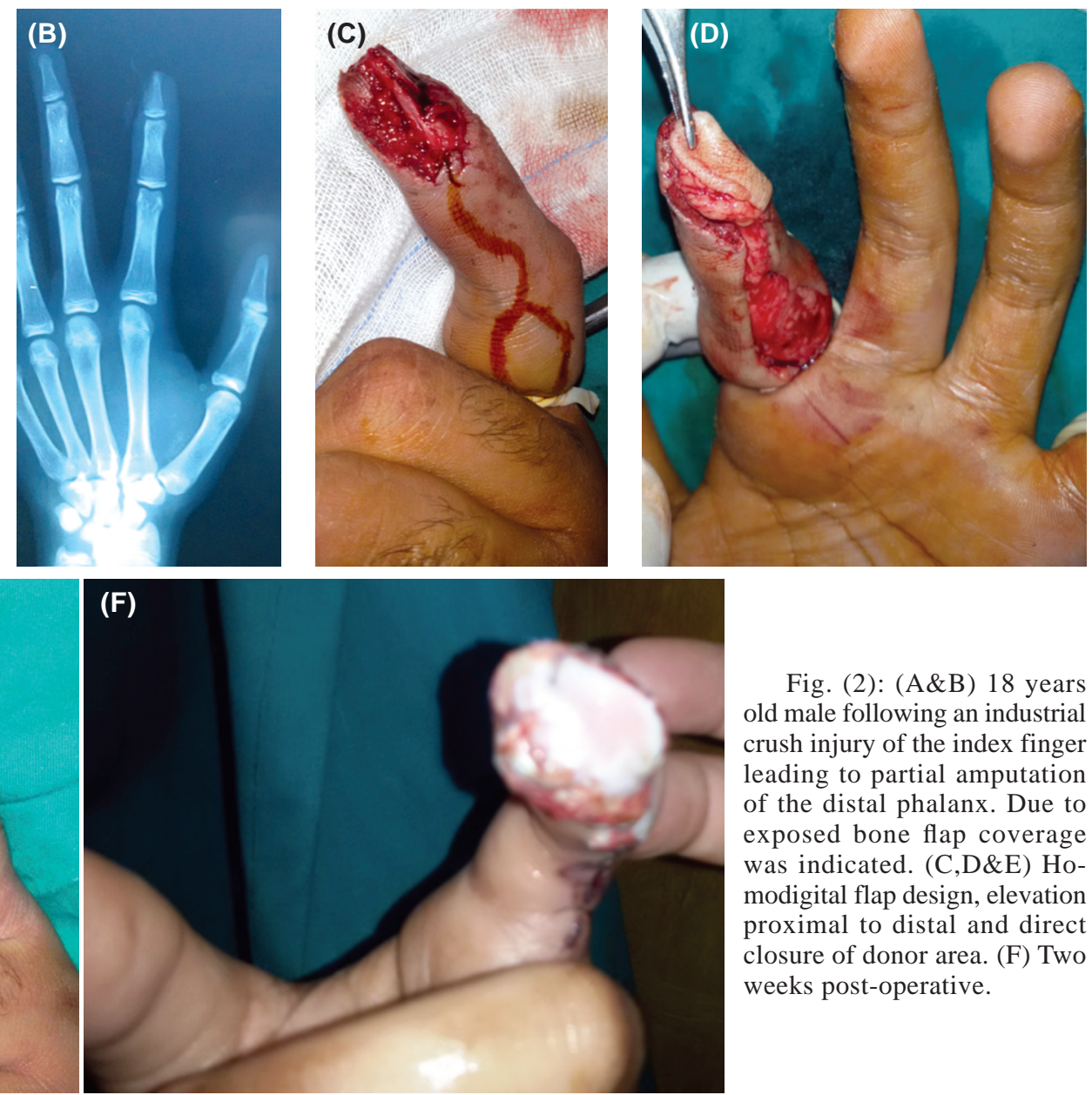

Fig. (2): (A\&B) 18 years old male following an industrial crush injury of the index finger leading to partial amputation of the distal phalanx. Due to exposed bone flap coverage was indicated. (C,D\&E) Homodigital flap design, elevation proximal to distal and direct closure of donor area. (F) Two weeks post-operative. 

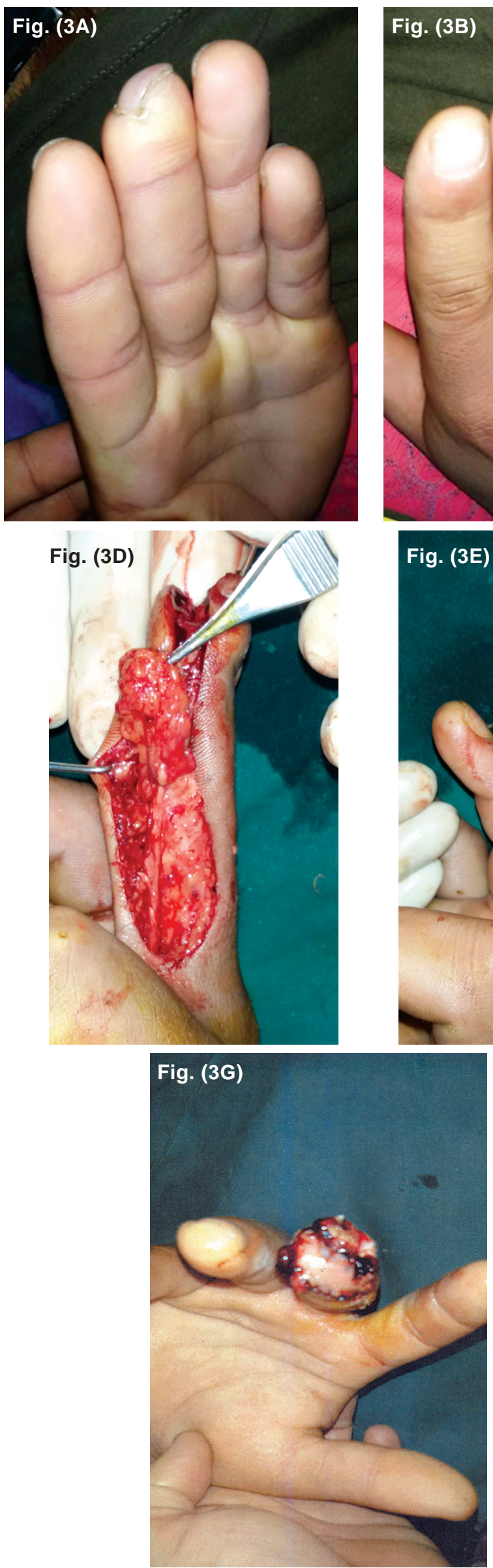
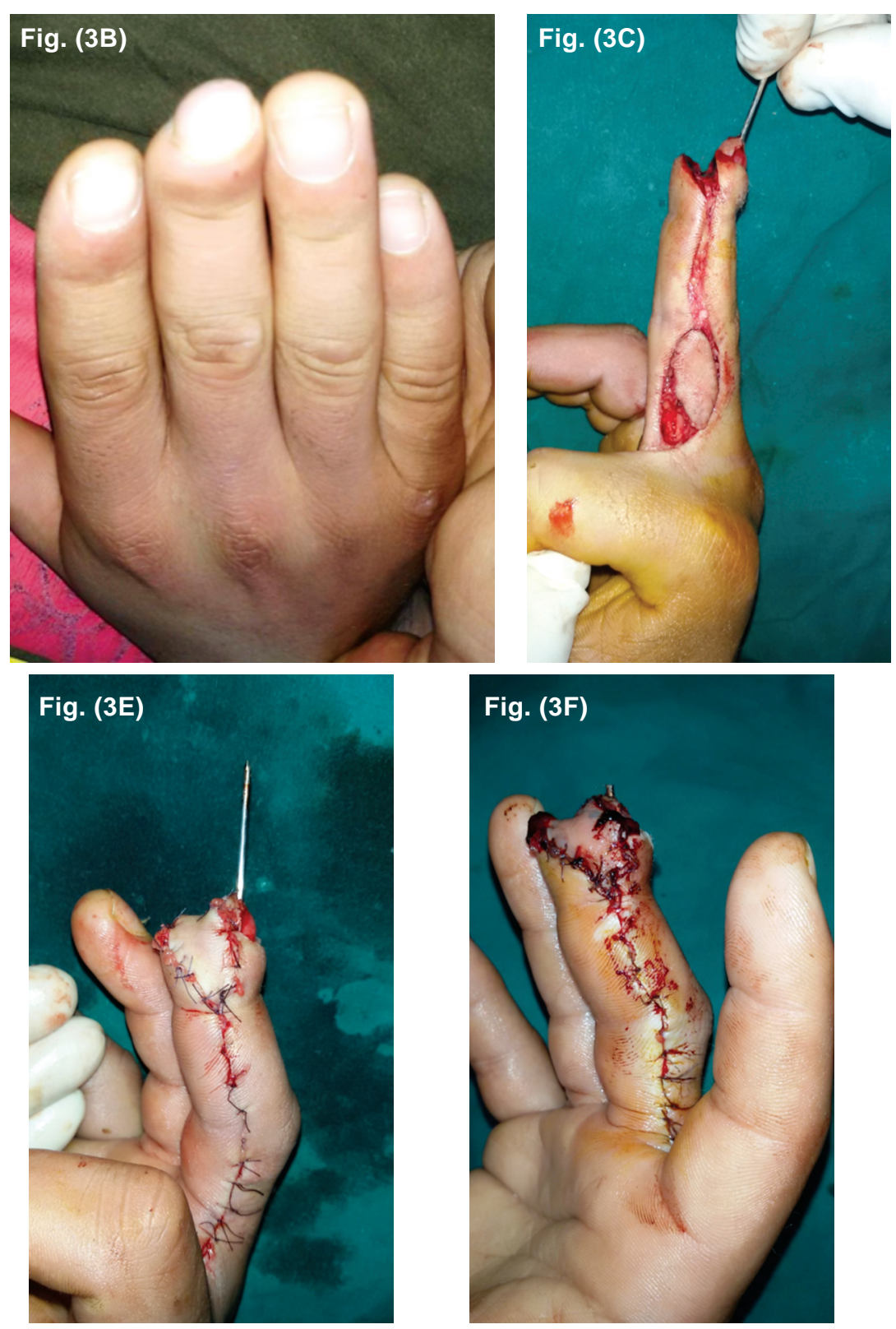

Fig. (3H)

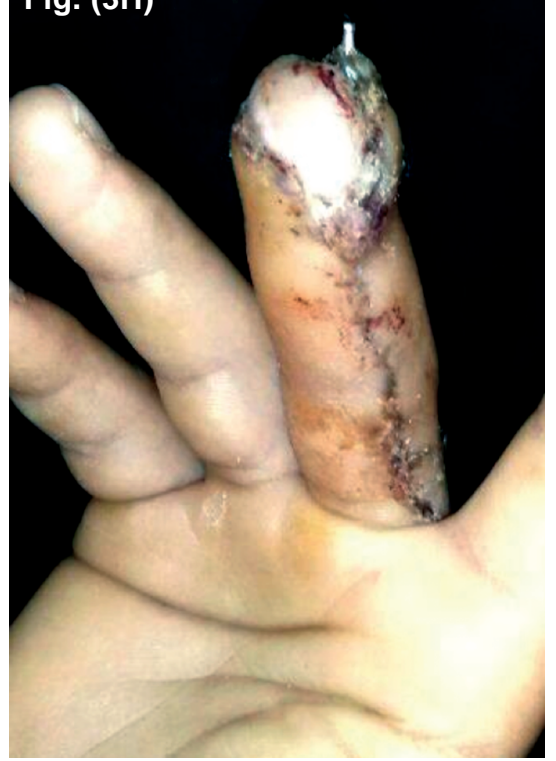



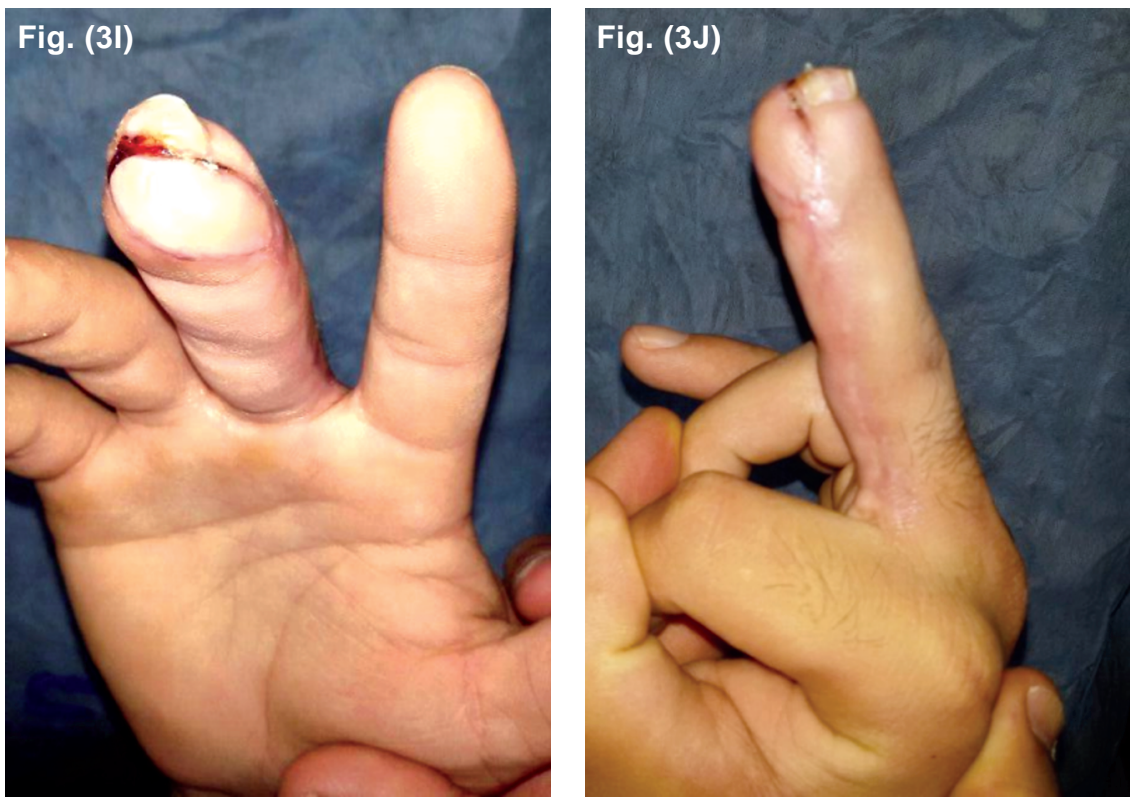

Fig. (3): (A\&B) 17 years old male with old trauma to distal phalanx right middle finger leading to obvious depression deformity due to tissue deficiency. (C,D\&E) Soft tissue release + $\mathrm{K}$. wire fixation and homodigital flap elevation. (F\&G) One week postoperative. (H) 2 weeks post-operative. (I\&J) One month post-operative.
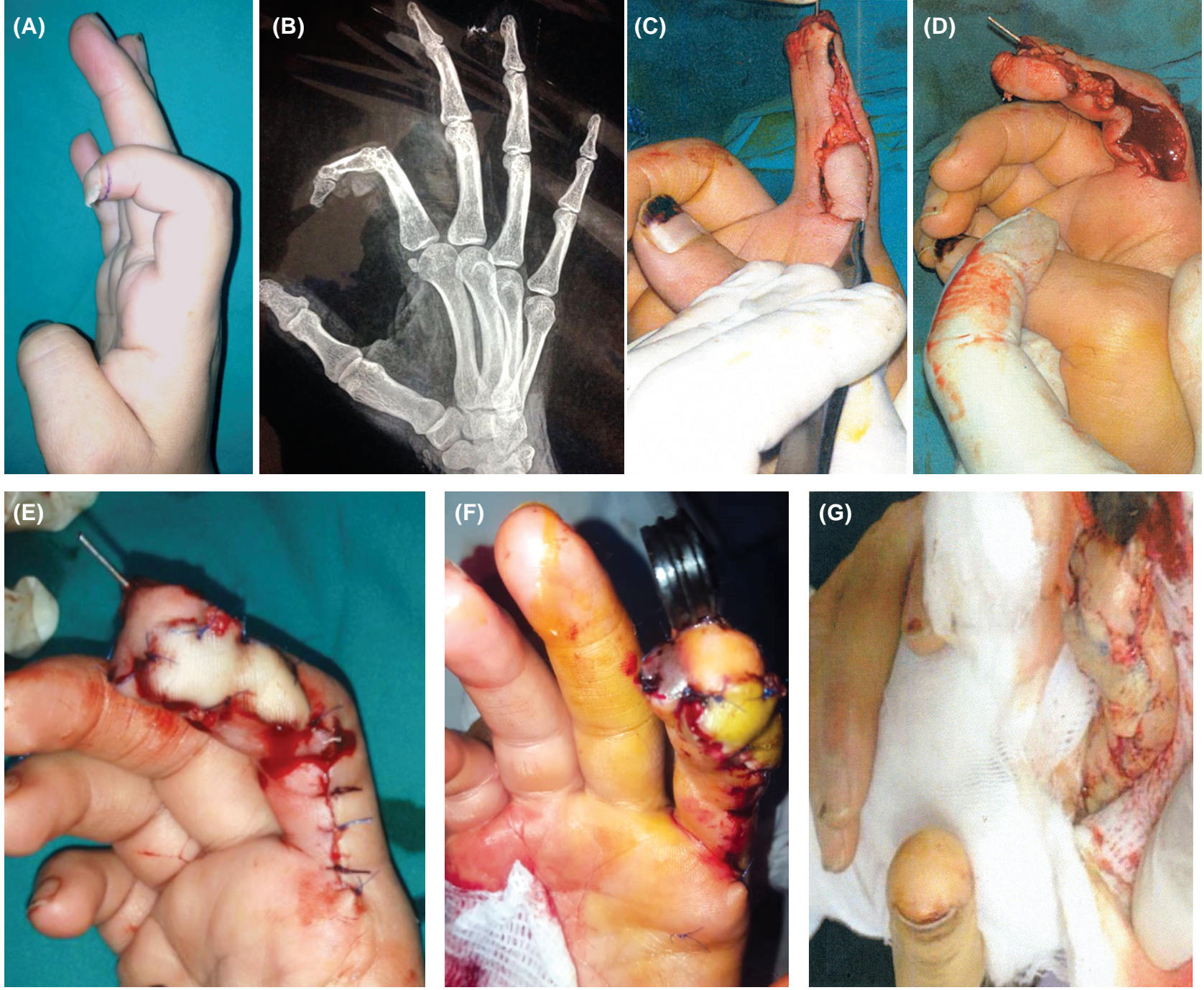

Fig. (4): (A\&B) 40 years old lady with painful parrot peak deformity of right index finger due to old trauma. (C,D\&E) Straitening of DIP + K. wire insertion, reversed island homodigital flap, SSG to cover pedicle and primary donor site closure. (F) $3^{\text {rd }}$ day post-operative, mild congestion. $(\mathrm{G})$ One week post-operative, note complete flap survival. 


\section{DISCUSSION}

Objectives of fingertip reconstruction include preservation of functional length and sensibility, prevention of symptomatic neuromas, maintenance of joint flexibility, acceptable donor site morbidity, absence of cold intolerance, minimal aesthetic deformity, single stage operation and quick return to work [12].

Other fingertip reconstruction tools include advancement flaps that often result in limitation in size and arc of rotation as well as cold intolerance. Regional flaps as thenar and cross finger flaps require 2 stage approach as well as the risk of joint stiffness specially in elderly. Free tissue transfer not only require microsurgical expertise but also subject the patient to prolonged surgery.

Weeks and Wray initially described the distally based homodigital island flap [13]. The technique was modified by Lia [14] and Kojima [6]. Momeni et al., evaluated the results of 11 cases reconstructed by the reversed homodigital island flap [12].

Advantages of the reversed homodigital island flap are that it allows a one stage operation avoiding a $2^{\text {nd }}$ operation (as seen in cross finger and thenar flaps) thus confining surgery to the involved digit only. It also avoids prolonged immobilization thus avoiding joint stiffness especially in the elderly. The glabrous skin of the lateral surface of the proximal phalanx allows reconstruction replacing like with like. The wide arc of rotation allows coverage of the most distal defects thus preserving functional length. Han et al., demonstrated no significant difference regarding 2 point discrimination between sensate and non-sensate flaps. This is due collateral sprouting from adjacent intact nerves with concomitant central adaptation mechanisms [10].

Disadvantages of the flap are that it is not suitable for defects proximal to distal interphalangeal joint due to injury of the distal transverse digital artery. Venous insufficiency is another problem with the flap. Sacrifice of one digital artery is another disadvantage.

\section{Conclusion}

Reverse homodigital island flap is a safe method for distal phalangeal reconstruction offering mul- tiple advantages over local, regional and free flaps with an almost negligible donor site defect.

\section{REFERENCES}

1- Gellman H.: Fingertip-nail bed injuries in children: Current concepts and controversies of treatment. J. Craniofac. Surg., 20: 1033-1035, 2009.

2- Hile D. and Hile L.: The emergent evaluation and treatment of hand injuries. Emerg. Med. Clin. North. Am., 33: 397408, 2015.

3- Elliot D. and Yii W.: Homodigital reconstruction of the digits: The perspective of one unit. Handchir. Mikrochir. Plast. Chir., 33 (1): 7-19, 2001.

4- Aboulwafa A. and Emara S.: Versatility of Homodigital Islandized Lateral VY Flap for Reconstruction of Fingertips and Amputation Stumps. Egypt. J. Plast. Reconstr. Surg., 37: 89-96, 2013.

5- Venkataswami R. and Subramanian N.: Oblique triangular flap: A new method of repair for oblique amputation of the fingertip and thumb. Plast. Reconstr. Surg., 66: 296$300,1980$.

6- Kojima T., Tsuchida Y., Hirase Y., et al.: Reverse vascular pedicle digital island flap. Br. J. Plast. Surg., 43: 290$295,1990$.

7- Lemmon J.A., Janis J.E. and Rohrich R.J.: Soft-tissue injuries of the fingertip: methods of evaluation and treatment. An algorithmic approach. Plast. Reconstr. Surg., 122: $105 \mathrm{e}-117 \mathrm{e}, 2008$.

8- Ozaksar K, Toros T. and Sugun T.S.: Reconstruction of finger pulp defects using homodigital dorsal middle phalangeal neurovascular advancement flap. J. Hand. Surg. Eur., 35: 125-129, 2010.

9- Iwasawa M., Kawamura T. and Nagai F.: Dorsally extended digital island flap for repairing soft tissue injury of the fingertip. J. Plast. Reconstr. Aesthet. Surg., 64: 13001305, 2011.

10- Han S.K., Lee B.I. and Kim W.K.: The reverse digital artery island flap: Clinical experience in 120 fingers. Plast. Reconstr. Surg., 101: 1006-11, 1998.

11- Ishikawa K., Ogawa Y., Soeda H., et al.: A new classification of the amputation level for the distal part of the fingers. J. Jpn. Soc. Microsurg., 3: 54-62, 1990.

12- Momeni A., Zajonc H., Kalash Z., et al.: Reconstruction of distal phalangeal injuries with the reverse homodigital island flap. Injury, 39 (12): 1460-3, 2008.

13- Weeks P.M. and Wray R.C.: Management of acute hand injuries: A biological approach. $2^{\text {nd }}$ ed., St Louis: Mosby Co., pp 140-143, 1973.

14- Lai C.S., Lin S.D., Chou C.K., et al.: Innervated reverse digital artery flap through bilateral neurorrhaphy for pulp defects. Br. J. Plast. Surg., 36: 483-488, 1993. 